\title{
28 Research Square \\ Fatal outcome in a COVID-19 patient despite IL 6 blockage in cytokine storm
}

\section{Michael Bovet ( $\nabla$ mbovet85@gmail.com )}

Klinik für Kardiologie, Pneumologie, Angiologie und Internistische Intensivmedizin (Medizinische Klinik B), Klinikum Ludwigshafen, Ludwigshafen, Germany

\section{Daniel Wadsack}

Klinik für Innere Medizin, Hämato-Onkologie, Nephrologie, Infektiologie und Rheumatologie

(Medizinische Klinik A), Klinikum Ludwigshafen, Ludwigshafen, Germany

\section{Florentina Kosely}

Klinik für Kardiologie, Pneumologie, Angiologie und Internistische Intensivmedizin (Medizinische Klinik B), Klinikum Ludwigshafen, Ludwigshafen, Germany

\section{Wolfgang Zink}

Klinik für Klinik für Anästhesiologie, Operative Intensivmedizin und Notfallmedizin, Klinikum Ludwigshafen, Ludwigshafen, Germany

\section{Ralf Zahn}

Klinik für Kardiologie, Pneumologie, Angiologie und Internistische Intensivmedizin (Medizinische Klinik B), Klinikum Ludwigshafen, Ludwigshafen, Germany

Keywords: COVID-19, SARS-CoV-2, cytokine storm, Interleukin 6, Tocilizumab, perimyocarditis, coagulopathy

Posted Date: May 7th, 2020

DOI: https://doi.org/10.21203/rs.3.rs-26470/v1

License: (c) (i) This work is licensed under a Creative Commons Attribution 4.0 International License. Read Full License 


\section{Abstract}

A 59 year old male patient was admitted to our hospital diagnosed with COVID-19 associated pneumonia. Upfront treatment with hydroxychloroquine and azithromycin was started. Because of clinical deterioration with ARDS, circulatory shock and increased hyperinflammatory markers six days later, a cytokine storm rose to the top of differential diagnosis and off-label treatment with IL 6 receptor antagonist Tocilizumab was initiated. Subsequently we observed a dramatic rise of D-dimers indicating coagulopathy. Perimyocarditis with severe cardiac arrhythmia occurred after a second dose was administered. Later the patient died due to multi organ failure. Exacerbation of cytokine storm following treatment with Tocilizumab could not be ruled out, despite a hitherto unreported relationship with COVID19.

\section{Case Report}

Late march 2020, a 59 year old male patient presented with flu-like symptoms and initial manifestation of atrial flutter. He reported a fever, muscle aches, chills, dry cough, fatigue and palpitation ongoing for 5 days. Dyspnea was explicitly denied. Pre-existing conditions were hypertension and glaucoma, BMI was $26,3 \mathrm{~kg} / \mathrm{m} 2$ (188 cm, $93 \mathrm{~kg})$, no history of smoking, no allergies. On examination he was still febrile $\left(38,3^{\circ} \mathrm{C}\right)$ and oxygen saturation was $93 \%$. Lab testing revealed a slight decrease in lymphocyte and platelet count and inflammatory markers were elevated (Table 1). Two pairs of blood cultures were taken, RSV and influenza were tested negative in a nasopharyngeal swab. Later he was diagnosed with COVID19 , confirmed by a positive PCR test obtained from a nasopharyngeal swab and CT scan revealed typical findings (Fig. 1a). After admission we initiated off label therapy with hydroxychloroquine and azithromycine. QTc interval was measured on a daily basis.

In the following days the general condition worsened tremendously: fever, oxygen demand and inflammation markers increased continuously. The follow up CT scan on day 6 showed remarkable progression of pulmonary inflammation (Fig. 1b) and the patient was transferred to our intensive care unit. HFNC was started upfront, but due to absence of substantial clinical improvement the patient was intubated and treated with low tidal pressure-controlled ventilation the next day, which worked seamlessly because of preserved lung compliance.

On day 10 , we noticed worsening of hypoxemia and the patient developed severe circulatory shock, requiring a norepinephrine drip. On the basis of persistent fever $>39^{\circ} \mathrm{C}$ and rising levels of inflammation markers (CRP, IL 6, Ferritin) but solely marginally elevated levels of PCT, we suspected cytokine storm. Accordingly, we decided to start treatment with IL 6 receptor antagonist Tocilizumab (TCZ, 600 mg i.v.). At the same time, broad spectrum antibiotics Piperacillin/Tazobactam) were given prophylactically. The clinical status deteriorated nonetheless and pronation was necessary in order to establish sufficient oxygenation (P/F ratio >150). The first lab control after TCZ treatment showed a dramatic rise of IL 6 and D-dimers. The next day, vasopressor support had to be increased and the patient developed hypercapnia (pCO2 $96 \mathrm{mmHg}$ ) despite an increased respiratory rate (24/min) and driving pressure (22 mbar). A 
second dose of TCZ (600 mg i.v.) was administered. Because of refractory respiratory failure venovenous ECMO therapy was provided in order to mitigate ventilator-induced lung injury. Shortly thereafter, hemodynamic relevant ventricular extrasystoles and ventricular tachycardia occurred, persisting even after repositioning of ECMO cannula and treatment with Amiodarone. Lactate levels were exploding. On the morning of day 12 , troponin levels peaked at $580.000 \mathrm{pg} / \mathrm{nl}$, even though bedside echocardiogram revealed preserved left ventricular function and there was no sign of right-heart overload. Around noon, the circulatory shock was refractory (systolic blood pressure $50 \mathrm{mmHg}$ ) to high doses of vasopressors and 12-lead ECG showed ubiquitous ST elevations (Fig 2b). Transportation to cardiac catheter laboratory was waived due to hemodynamic instability and we decided to initiate veno-arterial ECMO for temporary mechanical circulatory support and simultaneous extracorporeal gas exchange. Despite all efforts, the patient died the same day from multi-organ failure.

\section{Discussion}

Since late December 2019, the spread of the novel lunge disease COVID-19 was observed initially in Wuhan, China and subsequently all over the world. In the beginning of a SARS-CoV-2 infection, virus replication and a direct viral cytopathic effect in the upper respiratory tract lead to a flu-like disease. For the latter and more severe stage of the disease - characterized by acute respiratory distress syndrome (ARDS), septic shock and multi organ failure - a dysregulated immune response is held accountable. [1] Clinical presentation and laboratory findings of uncontrolled activation of immune cells are similar to other cytokine release syndromes (CRS) like secondary hemophagocytic lymphohistiocytosis (sHLH) or CRS secondary to CAR T-cell therapy. In contrast to these entities, the immunopathology of COVID-19 is more focused on the lung, e.g. due to overactivity of alveolar cell macrophages. [1, 2] Hereby, elevated IL 6 levels have been identified as a prognostic marker for severe outcomes of COVID-19 and may play a pivotal role in pathogenesis of ARDS. [3,4] No therapies have been shown effective to date. [5] In a retrospective analysis, IL 6 receptor antagonist tocilizumab (TCZ) has shown to be a potential and promising treatment approach: In a Chinese study, twenty-one patients had been treated with a single dose of $400 \mathrm{mg}$ to suppress a cytokine storm of the host and clinical and radiological features improved consecutively. [6, 7] An additional single-center analysis of 15 patients also reported mainly positive results. [8] Following these results, more than ten randomized controlled trials (RCTs) have been initiated, with results pending to the time of this report. According to Chinese Guideline of Diagnosis and treatment of COVID-19 (7th edition, April 2020) the application of 4-8 mg/kg should be considered in patients with extensive lung involvement and high IL 6 levels. TCZ is an approved treatment option for high inflammatory systemic diseases like rheumatoid arthritis and CRS secondary to CAR T-cell therapy. The inhibition of IL 6 , a central proinflammatory cytokine, results in suppression of the downstream signaling pathway and thus normalization of inflammatory markers such as CRP. Toxicity rates of this treatment are low, most frequently elevated transaminases, hyperlipidemia, neutropenia, diarrhea and bacterial infections (namely diverticulitis) are observed. [9]

Discriminating between cytokine storm, sepsis and ARDS without hyperinflammatory dysregulation is rather difficult. In this case we assumed a cytokine storm due to following findings: ARDS, hypotension, 
sterile blood cultures, persisting fever $>39^{\circ} \mathrm{C}$, high levels of CPR, ferritin and IL 6 despite low levels of PCT. Therefore, we decided to administer a dose of $600 \mathrm{mg} \mathrm{TCZ}$ with an optional second dose in absence of clinical improvement.

The first lab test after administration showed a dramatic rise in IL 6 levels, which is frequently observed after application of TCZ in other entities due to a reduced clearance of IL 6 through TCZ/IL 6 receptor complex. Hence, the surge reflects the true disease activity and rather not a compensation mechanism. [10] A detrimental effect is unlikely, but possible. This initial rise of IL 6 has been confirmed in another analysis in the majority of COVID-19 patients treated with TCZ. [8] Additionally, a tremendous increase in D-dimers and fibrin monomers occurred, indicating development of coagulopathy. Clinical signs of disseminated intravascular coagulation were absent (ISTH scoring system for DIC: 3 points). In general, a relevant proportion of COVID-19 patients feature early stages of coagulopathy presenting elevated levels of fibrin degradation products (D-dimers, fibrin monomers), which are associated with thromboembolic complications and poor outcome. $[11,12]$ The relationship of hyperinflammation and coagulopathy is well established in septic shock from various infectious agents and is also assumed for SARS-CoV-2 infection. In clinical examination of our patient, neither DVT, pulmonary embolism nor arterial thrombotic event were observed. Nevertheless, it is possible that clotting of microcirculation may be a cause for elevated levels of lactate and worsening hypoxemia: examination of deceased COVID-19 patients demonstrated clotting of the pulmonary microcirculation, indicating a limited coagulopathy of the lung. $[1,13]$

After the second administration of TCZ, malignant cardiac arrhythmia, a massive rise of troponin levels and ubiquitous ST-elevations occurred. Cardiac arrhythmia is frequently seen in severe COVID-19 cases (VT/VF in $6 \%$ of those cases) and elevated levels of troponin are also associated with poor outcome. [14] The underlying mechanism of cardiac injury is poorly understood. Recently, two patients with systolic dysfunction due to COVID-19 have been described, with progressive clinical stabilization and improved left ventricular function after immunosuppressive therapy. $[15,16]$ This has been interpreted as a direct connection between hyperinflammation and myocarditis as it has been proven before during the H1N1 pandemic. [17] Accordingly, we suspected a COVID-19 induced inflammatory perimyocarditis. Obstructive coronary artery disease seemed unlikely due to preserved left ventricular function and abnormalities in ECG leads that did not match a coronary territory. [18]

Finally, it was not possible to discriminate if the rapid worsening clinical condition was a result of COVID19 itself or if our therapy contributed to this deterioration. To our knowledge, there are no reports observing comparable grave side effects of TCZ in COVID-19 - especially regarding an exacerbation of a preexisting cytokine storm as we considered possible. Solely one case report described a similar clinical aggravation of coagulopathy in a patient receiving TCZ after developing CRS secondary to CAR-T cell therapy and bacterial superinfection was suspected to be responsible. [19] In our patient PCT levels were normal before both administrations and broad spectrum antibiotic was applied prophylactically.

Nevertheless, septic shock due to fungal pneumonia cannot be ruled out. Furthermore, discussing others factors leading to elevated inflammation markers is crucial. Several studies have shown that mechanical 
ventilation itself increases proinflammatory cytokines. [20] In order to classify such dramatic events after TCZ treatment, the pending results of ongoing RCTs are absolutely essential. Until then, off-label use of TCZ in COVID-19 should only be considered after thorough weighing of all available information and explicit consultation about severe side effects.

\section{Conclusion}

- Currently there is no approved or clearly efficacious treatment option for COVID-19

- ARDS, septic shock, thromboembolic and cardiac events are responsible for fatal outcomes, presumably due to dysregulation of immune response (cytokine storm)

- Based on our experience, deterioration and exacerbation of cytokine storm triggered by IL 6 receptor antagonist Tocilizumab cannot be ruled out

- Randomized and placebo controlled trials are crucial to investigate efficacy and safety for Tocilizumab as treatment for COVID-19

\section{Abbreviations}

DIC: disseminated intravascular coagulation

HFrEF: heart failure with reduced ejection fraction

HFNC: high flow nasal cannula
ISTH: International Society on Thrombosis and Haemostasis

DVT: deep vein thrombosis

VT/VF: ventricular tachycardia/fibrillation

\section{Declarations}

Corresponding author: Dr. Michael Bovet, Klinik für Kardiologie, Pneumologie, Angiologie und Internistische Intensivmedizin (Medizinische Klinik B), Klinikum Ludwigshafen, Ludwigshafen, Germany Germany. E-mail: mbovet85@gmail.com

Conflicts of interest: The authors declare that they have no conflict of interest.

Funding: No funding applicable to this article

Consent: Written informed consent was obtained from the patient's family for publication of this case report.

\section{References}


1. McGonagle, D., et al., The Role of Cytokines including Interleukin-6 in COVID-19 induced Pneumonia and Macrophage Activation Syndrome-Like Disease. Autoimmunity Reviews, 2020: p. 102537.

2. Chaofu Wang, J.X., Lei Zhao et al., Aveolar Macrophage Activation and Cytokine Storm in the Pathogenesis of Severe COVID-19. PREPRINT (Version 1) available at Research Square, 2020.

3. Ruan, Q., et al., Clinical predictors of mortality due to COVID-19 based on an analysis of data of 150 patients from Wuhan, China. Intensive Care Med, 2020.

4. Meduri, G.U., et al., Inflammatory cytokines in the BAL of patients with ARDS. Persistent elevation over time predicts poor outcome. Chest, 1995. 108(5): p. 1303-14.

5. Sanders, J.M., et al., Pharmacologic Treatments for Coronavirus Disease 2019 (COVID-19): A Review. JAMA, 2020.

6. Xu X, H.M., Li T, Sun W, Wang D, Fu B, et al. , Effective treatment of severe COVID-19 patients with tocilizumab. ChinaXiv:202003000026, 2020.

7. Zhang, C., et al., The cytokine release syndrome (CRS) of severe COVID-19 and Interleukin-6 receptor (IL-6R) antagonist Tocilizumab may be the key to reduce the mortality. Int J Antimicrob Agents, 2020: p. 105954.

8. Luo, P., et al., Tocilizumab treatment in COVID-19: A single center experience. J Med Virol, 2020.

9. Mohan, S., et al., OP0105 Long-term safety of tocilizumab from large clinical trial and postmarketing populations. Vol. 76. 2017. 95.2-95.

10. Nishimoto, N., et al., Mechanisms and pathologic significances in increase in serum interleukin-6 (IL6) and soluble $I L-6$ receptor after administration of an anti-IL-6 receptor antibody, tocilizumab, in patients with rheumatoid arthritis and Castleman disease. Blood, 2008. 112(10): p. 3959-3964.

11. Klok, F.A., et al., Incidence of thrombotic complications in critically ill ICU patients with COVID-19. Thrombosis Research, 2020.

12. Wu, C., et al., Risk Factors Associated With Acute Respiratory Distress Syndrome and Death in Patients With Coronavirus Disease 2019 Pneumonia in Wuhan, China. JAMA Internal Medicine, 2020.

13. Luo, W.Y., H.; Gou, J.; Li, X.; Sun, Y.; Li, J.; Liu, L. , Clinical Pathology of Critical Patient with Novel Coronavirus Pneumonia (COVID-19). Preprints 2020, 2020020407, 2020.

14. Lazzerini, P.E., M. Boutjdir, and P.L. Capecchi, COVID-19, Arrhythmic Risk and Inflammation: Mind the Gap! Circulation, 2020.

15. Hu, H., et al., Coronavirus fulminant myocarditis treated with glucocorticoid and human immunoglobulin. European Heart Journal, 2020.

16. Inciardi, R.M., et al., Cardiac Involvement in a Patient With Coronavirus Disease 2019 (COVID-19). JAMA Cardiology, 2020.

17. Ukimura, A., et al., A National Survey on Myocarditis Associated With the 2009 Influenza A (H1N1) Pandemic in Japan. Circulation Journal, 2010. 74(10): p. 2193-2199.

18. Bangalore, S., et al., ST-Segment Elevation in Patients with Covid-19 - A Case Series. N Engl J Med, 2020. 
19. Liu, Y., et al., Hemofiltration Successfully Eliminates Severe Cytokine Release Syndrome Following CD19 CAR-T-Cell Therapy. J Immunother, 2018. 41(9): p. 406-410.

20. Maia, L.A., et al., Effects of Protective Mechanical Ventilation With Different PEEP Levels on Alveolar Damage and Inflammation in a Model of Open Abdominal Surgery: A Randomized Study in Obese Versus Non-obese Rats. Front Physiol, 2019. 10: p. 1513.

\section{Table}

Due to technical limitations Table 1 is available as a download in the Supplementary Flles.

\section{Figures}

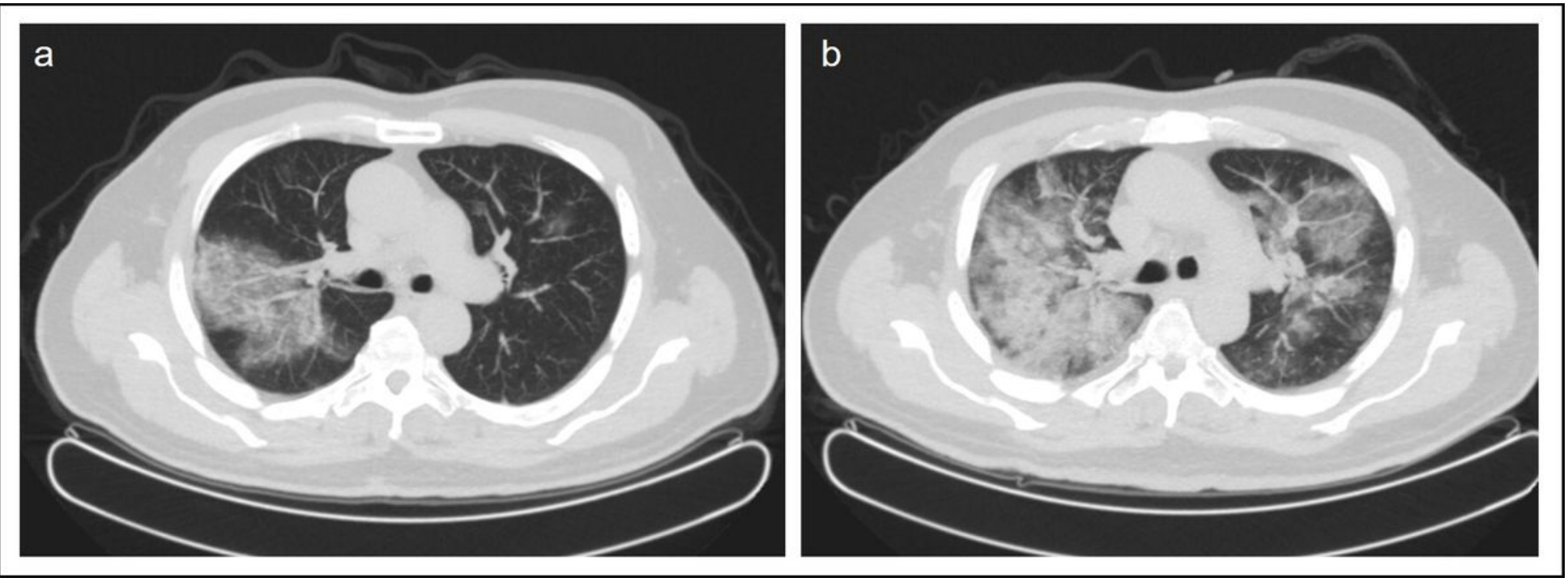

\section{Figure 1}

a Chest CT scan on the day of hospital admission showing bilateral and peripheral ground glass opacities. b Considerable progression on day 6 with predominantly consolidated infiltrates in all lung lobes. 


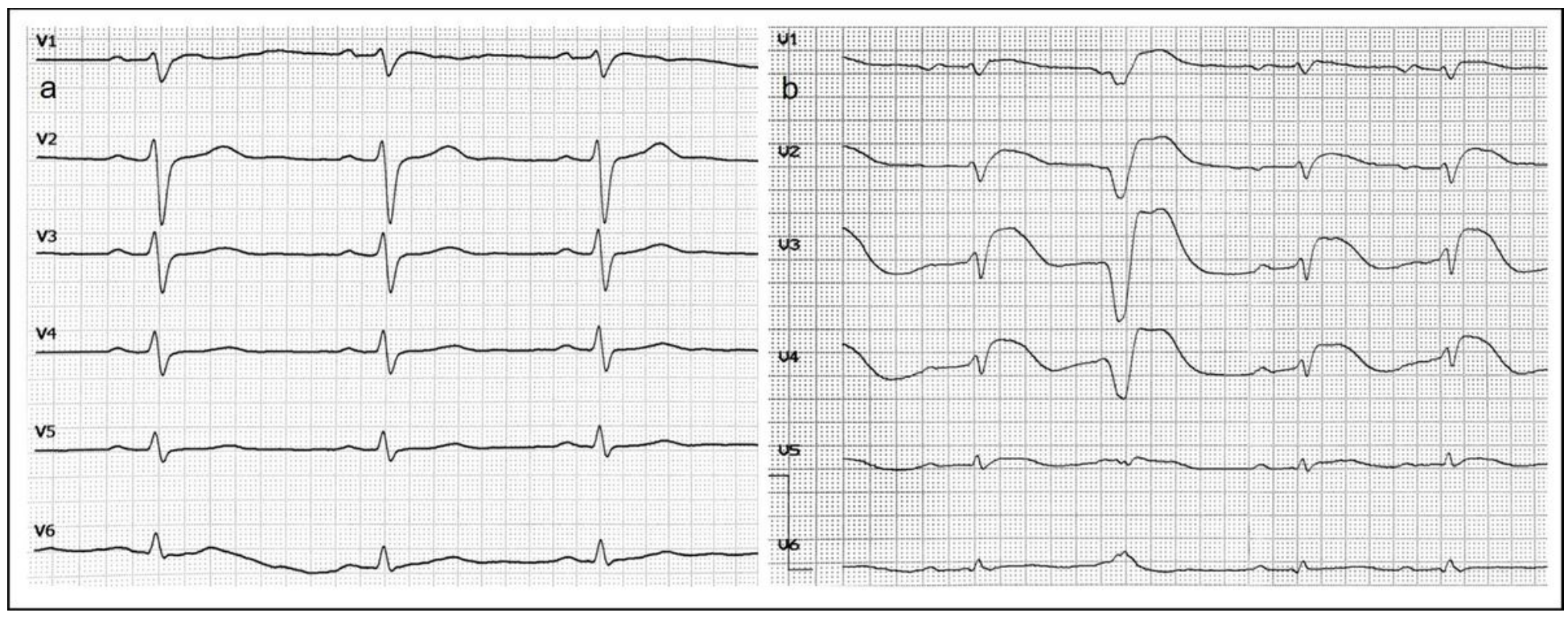

Figure 2

a ECG on the day of admission without ST-elevations. b ubiquitous ST-elevations and multiple ventricular extrasystoles on day 12 (no presentation of limb leads in this figure).

\section{Supplementary Files}

This is a list of supplementary files associated with this preprint. Click to download.

- Table1.pdf 